\title{
Resource Optimization of Spatial TDMA in Ad Hoc Radio Networks: A Column Generation Approach
}

\author{
Patrik Björklund, Peter Värbrand and Di Yuan \\ Department of Science and Technology, Linköping University \\ SE-601 74 Norrköping, Sweden \\ patbj, petva, diyua@itn.liu.se
}

\begin{abstract}
Wireless communications using ad hoc networks are receiving an increasing interest. The most attractive feature of ad hoc networks is the flexibility. The network is set up by a number of units in an ad hoc manner, without the need of any fixed infrastructure. Communication links are established between two units if the signal strength is sufficiently high. As not all pairs of nodes can establish direct links, the traffic between two units may have to be relayed through other units. This is known as the multi-hop functionality.

Design of ad hoc networks is a challenging task. In this paper we study the problem of resource allocation with spatial TDMA (STDMA) as the access control scheme. Previous work for this problem has mainly focused on heuristics, whose performance is difficult to analyze when optimal solutions are not known. We develop, for both node-oriented and link-oriented allocation strategies, mathematical programming formulations for resource optimization. We further present a column generation approach, which, in our numerical experiments, constantly yields optimal or near-optimal solutions. Our results provide important benchmarks when evaluating heuristic on-line algorithms for resource optimization using STDMA.
\end{abstract}

Index Terms - Ad hoc networks, STDMA, node and link assignment, column generation

\section{INTRODUCTION}

An ad hoc network is characterized by a collection of radio units with a wireless interface forming temporary connections. No fixed infrastructure is involved in the communication. Instead, two radio units can establish a direct communication link, if the signal-to-noise ratio is high enough. Two radio units far away from each other may communicate, if the units between them are participating in the ad hoc network, and are willing to forward packets for them (so called the multi-hop functionality).

Multi-hop radio networks have mainly been considered for military command and control systems, because a centralized network is often not feasible in such applications. However, in recent years there is a growing interest in ad hoc networks in other applications, such as peer-to-peer computer communications, and communications between mobile sensors (e.g. traffic safety systems).

As pointed out in [9], ad hoc networks pose many design challenges. In this paper, we address the issue of resource allocation when designing link access schemes. One access scheme for ad hoc networks is Time Division Multiple Access (TDMA), in which the transmission resource of a radio frequency is divided into time slots, and a unit may transmit in one or several time slots. It is known that, although simple to implement, TDMA is very inefficient from the resource utilization point of view. One possibility to increase the network efficiency is to use Spatial TDMA, or STDMA [16], which takes into account that radio units are usually spread out geographically, and hence units with a sufficient spatial separation can use the same time slot for transmission.

A number of specific planning problems, with different levels of complexity, can be defined for access control schemes in ad hoc networks. For example, one of the most challenging problems is to design efficient, distributed algorithms that can take traffic information into account, and can handle fully mobile scenarios. Here, we will address the problem of computing STDMA slot allocation centrally for static networks without any knowledge of the traffic distribution. This is one of the most basic planning problems in this area. The objective of the optimization problem is to minimize the length of the STDMA data frame, such that all the units (or all the network links) are assigned at least one time slot. Previous work for this problem (e.g. [4], [6], [11], [14], and [17]) has focused on heuristics. There is, therefore, a lack of methods that aim at finding optimal solutions, which are important both for evaluating heuristic algorithms, and for assessing the true potential of STDMA. The main purpose of this paper is to present set covering formulations to model resource optimization problems for both node-oriented and link-oriented allocation strategies, and an efficient column generation solution method. The proposed method generates optimal or near-optimal solutions in our numerical experiments. The focus of this paper is not the methodology itself, but its application in context of ad hoc networks. Up to our knowledge, such an investigation has not been conducted previously. We show that, in addition to theoretical analyzes of ad hoc network capacities (such as [19]), mathematical programming methodologies 
can aid as a powerful tool in rational ad hoc network design. Indeed, the results from this research can be used as reference solutions when the complexity is increased, and more realistic situations are tackled. Some of these future research ideas are discussed in Section VIII.

The remainder of this paper is organized as follows. In Section II we discuss two strategies of resource allocation. The optimization problems are formalized in Section III, and the computational complexity is studied in Section IV. We present mathematical formulations in Section V, and the column generation method in Section VI. Numerical results are presented in Section VII, and conclusions are drawn in Section VIII.

\section{Assignment Strategies}

Resource allocation in STDMA can be node-oriented (node assignment) or link-oriented (link assignment). In the former strategy, a node is assigned one or several time slots. In each of these time slots, the node may use any of its links for transmitting data to another node. To avoid access collisions, the nodes close to a transmitting node are not allowed to transmit in the same time slot. In addition, when a node is transmitting, it is required that the accumulated interference on any of the outgoing links does not exceed a certain threshold. It can be realized that this assignment strategy is well-suited for broadcast traffic.

In link assignment, a link is assigned one or several time slots for communications between a specific pair of nodes (point-to-point). Considerations for avoiding access collisions and interference are similar to those for node assignment. Generally speaking, link assignment achieves a higher spatial reuse of the time slots, but is, however, less suitable for broadcast traffic. STDMA resource allocation based on node assignment has been studied in [2], [4], [5], and [6], while [3], [10], [14], and [17] focus on algorithms using link assignment. Comparisons of the two assignment strategies can be found in [11], [12], [13], and [18].

\section{PRoblem Definitions}

We will use the assumptions and notation in [10] when defining the optimization problems. Consider an ad hoc network consisting of a set of nodes $N$, where each node $i \in N$ represents a radio unit. A direct communication link can be established between nodes $i$ and $j$ if the corresponding signal-to-noise ratio (SNR) is greater than or equal to a certain threshold, that is, if

$$
\operatorname{SNR}(i, j)=\frac{P_{i}}{L_{b}(i, j) N_{r}} \geq \gamma_{0},
$$

where $P_{i}$ is the transmitting power of $i, L_{b}(i, j)$ is the path-loss between $i$ and $j$, and $N_{r}$ is the effect of the thermal noise. We use $A$ to denote the set of (directed)

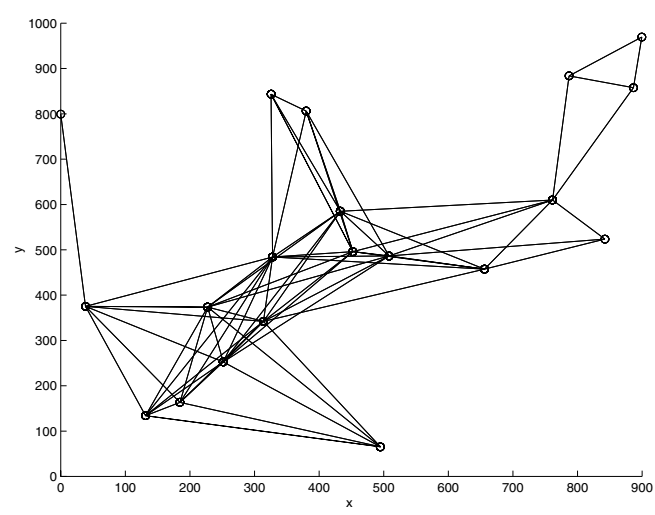

Fig. 1. An ad hoc network with 20 nodes.

links. It has been commonly assumed (e.g., [12] and [13]) that $P_{i}=P, \forall i \in N$, and $L_{b}(i, j)=L_{b}(j, i), \forall(i, j) \in A$. We do not make these assumptions in this paper, because our mathematical models and solution algorithms apply also to the general case.

As the nodes are usually out-spread, an ad hoc network is typically sparsely connected. A sample network of 20 nodes is shown in Figure 1.

There are several constraints and restrictions when assigning the STDMA time slots. First, a node can only transmit or receive, but not both, in a time slot. Secondly, a node can only receive data from one other node at a time, and finally, a link is error-free only if the signalto-interference ratio (SIR) is greater than or equal to a threshold. For link $(i, j)$, the last criterion can be expressed as

$$
\operatorname{SIR}(i, j)=\frac{P_{i}}{L_{b}(i, j)\left(N_{r}+\sum_{k \in N, k \neq i, j} \frac{P_{k}}{L_{b}(k, j)}\right)} \geq \gamma_{1}
$$

where the term $\sum_{k \in N, k \neq i, j} \frac{P_{k}}{L_{b}(k, j)}$ is the accumulated interference from other nodes.

The optimization problems for node assignment and link assignment have the same input, namely a set of nodes $N$, the path-loss between every pair of nodes $L_{b}(i, j)$, the transmitting power $P_{i}$ of each node, the noise effect $N_{r}$, and the two threshold values $\gamma_{0}$ and $\gamma_{1}$. The constraints for the node assignment problem are the following.

- Two nodes that are connected by a link must be assigned different time slots.

- Two nodes, both having directed links to a third node, must be assigned different time slots.

- A time slot can be assigned to a node only if all the outgoing links of the node satisfy the SIR-constraints (2).

The objective of the node assignment problem is to assign at least one time slot to each node, such that the total number of time slots is minimized. 
For link assignment, the corresponding constraints are the following.

- Two links that are adjacent, irrespective of the link directions, must be assigned different time slots.

- A time slot can be assigned to a link only if the SIRconstraint (2) for the link is satisfied.

The objective of the link assignment problem is to assign at least one time slot to each link, such that the total number of time slots is minimized.

\section{Computational Complexity}

We are able to show the following results for the optimization problems defined in the previous section.

Proposition 1 The node assignment problem is NPhard.

Proof Consider the graph coloring problem defined for an undirected graph $G=(V, E)$. We construct, in polynomial time, an instance of the node assignment problem, such that the two problems are equivalent. First, for each edge $(i, j) \in E$, we introduce a node $v_{i j}$. Let $V_{E}=\left\{v_{i j}, \forall(i, j) \in E\right\}$. We then let $N=V \cup V_{E}$. The set of directed links between the nodes in $N$, is defined as following. For a node $v_{i j} \in V_{E}$, we define two directed links, $\left(i, v_{i j}\right)$ and $\left(j, v_{i j}\right)$, and for every pair of nodes $v_{i j} \in V_{E}$ and $v_{k l} \in V_{E}$, we defined a pair of directed links, $\left(v_{i j}, v_{k l}\right)$ and $\left(v_{k l}, v_{i j}\right)$. We use $A$ to denote the set of directed links.

We let $\gamma_{0}=\gamma_{1}=1, P_{i}=2, \forall i \in N$, and $N_{r}=1$. For each link $(i, j) \in A$, we let $L_{b}(i, j)=1$. For all the pairs of nodes that do not have a link in $A$, we choose a sufficiently large path-loss value, such that the SNR constraint for these node pairs are not satisfied, and in addition, the SIR constraints for links in $A$ are redundant. (One pathloss value that achieves this is $2|N|$.) We then obtain an instance for the node assignment problem, defined for the node set $N$ and link set $A$, along which the other problem parameters defined as above.

To see the equivalence between the original node coloring problem and the derived node assignment problem, we make the following observations. First, the nodes in set $V_{E}$ must be assigned different time slots (or colors). Secondly, the node sets $V$ and $V_{E}$ must use disjoint sets of colors. Finally, two adjacent nodes in the graph coloring problem cannot use the same time slot. These observations lead directly to the conclusion that a feasible solution of one problem corresponds to a feasible solution of the other problem, and vice versa. For any of such pairs of solutions, the difference in the objective values is a constant (equals $|E|$ ). We further note that the reduction itself is polynomial. Hence the conclusion.

Proposition 2 The link assignment problem is NP-hard.

Proof Consider the edge coloring problem defined for a undirected graph $G=(V, E)$ (known to be NP-hard). We construct, in polynomial time, an instance of the link assignment problem, such that the two problems are equivalent. For each edge $(i, j) \in E$, we define a direct link from $i$ to $j$. Denote the set of directed links by $A$. We further let $N=V$. We then choose the values of transmitting power, the noise effect, the path-loss parameters, and the threshold values as in the previous proof. It is easily realized that the derived link assignment problem is equivalent to the edge coloring problem, and the conclusion follows immediately.

\section{Mathematical Formulations}

\section{A. A Node-Slot Formulation}

In this section, we present a node-slot formulation for optimally assigning the time slots to the nodes. We use $T=\{1, \ldots,|T|\}$ to denote a set of time slots. To ensure feasibility, it is sufficient to have $|T|=|N|$. We introduce the following variables.

$$
\begin{aligned}
& x_{i t}= \begin{cases}1 & \text { if time slot } t \text { is assigned to node } i \\
0 & \text { otherwise }\end{cases} \\
& y_{t}= \begin{cases}1 & \text { if time slot } t \text { is used } \\
0 & \text { otherwise }\end{cases}
\end{aligned}
$$

The node-slot formulation (NSP) for STDMA node assignment is

$$
\begin{aligned}
{[\mathrm{NSP}] \quad } & \min \sum_{t \in T} y_{t} \\
& \sum_{t \in T} x_{i t} \geq 1, \forall i \in N, \\
& x_{i t} \leq y_{t}, \forall i \in N, \forall t \in T, \\
& x_{i t}+\sum_{j:(j, i) \in A} x_{j t} \leq 1, \forall i \in N, \forall t \in T, \\
& \frac{P_{i} / N_{r}}{L_{b}(i, j)} x_{i t}+\gamma_{1}\left(1+M_{i j}\right)\left(1-x_{i t}\right) \geq \gamma_{1}(1+ \\
& \left.\sum_{k \in N: k \neq i, j} \frac{P_{k} / N_{r}}{L_{b}(k, j)} x_{k t}\right), \forall(i, j) \in A, \forall t \in T, \\
& x_{i t} \in\{0,1\}, \forall(i, j) \in A, \forall t \in T, \\
& y_{t} \in\{0,1\}, \forall t \in T .
\end{aligned}
$$

Here, the objective function (3) minimizes the total number of assigned time slots. Constraints (4) ensure that every node is assigned at least one time slot, and (5) are the coupling constraints between the two sets of variables. Constraints (6) ensure that different time slots are assigned to two nodes if they are connected by a link, or if they both have links to a third node. The SIR-constraints are defined in (7). Note that, if time slot $t$ is not assigned to node $i$, i.e., $x_{i t}=0$, then $(7)$ is redundant for a sufficiently large value of $M_{i j}$. Otherwise, the constraint becomes

$$
\frac{P_{i} / N_{r}}{L_{b}(i, j)} \geq \gamma_{1}\left(1+\sum_{k \in N: k \neq i, j} \frac{P_{k} / N_{r}}{L_{b}(k, j)} x_{k t}\right),
$$


which corresponds to (2).

The coefficient $M_{i j}$ can be set to $M_{i j}=$ $\sum_{k \in N: k \neq i, j}\left(P_{k} / N_{r}\right) / L_{b}(k, j)$, i.e., the sum of interference from all other nodes than $i$ and $j$. However, not all of the potential interfering nodes will be transmitting at the same time, because the slot assignment of these nodes must also satisfy constraints (6) and (7). Therefore, it is possible to calculate a smaller value of $M_{i j}$ in order to improve the LP-relaxation of NSP (we refer to [1] for details).

One may also note that the formulation NSP contains a lot of symmetry. For instance, there are many solutions corresponding to the same assignment, but with different time slots allocated. To break such symmetry, one may add the constraints $y_{t} \leq y_{t-1}, t=2, \ldots,|T|$, which imply that time slot $t$ is assigned only if time slot $t-1$ is assigned. Another type of symmetry is related to the fact that swapping the nodes of any two time slots does not affect the objective function value. One possibility to handle this is to require that the slot assigned to node $i$ has an index less or equal to $i$, which can be stated as $x_{i t}=0, \forall i, t: i<t$.

\section{B. A Link-Slot Formulation}

For link assignment, there is a link-slot formulation, LSP, which is similar to NSP. In LSP, the cardinality of the set of time slots can be set to the number of links (i.e., $|T|=|A|)$. We use the following variables in LSP.

$x_{i j t}= \begin{cases}1 & \text { if time slot } t \text { is assigned to link }(i, j), \\ 0 & \text { otherwise. }\end{cases}$

$y_{t}= \begin{cases}1 & \text { if time slot } t \text { is used } \\ 0 & \text { otherwise. }\end{cases}$

$v_{i t}= \begin{cases}1 & \text { if node } i \text { is transmitting in time slot } t \\ 0 & \text { otherwise. }\end{cases}$

Formulation LSP is stated below.

$$
\begin{aligned}
\text { [LSP] } & \min \sum_{t \in T} y_{t} \\
& \sum_{t \in T} x_{i j t} \geq 1, \forall(i, j) \in A \\
& x_{i j t} \leq y_{t}, \forall(i, j) \in A, \forall t \in T \\
& \sum_{j:(i, j) \in A} x_{i j t}+\sum_{j:(j, i) \in A} x_{j i t} \leq 1, \\
& x_{i j t} \leq v_{i t}, \forall(i, j) \in A, \forall t \in T, \forall t \in T \\
& \frac{P_{i} / N_{r}}{L_{b}(i, j)} x_{i j t}+\gamma_{1}\left(1+M_{i j}\right)\left(1-x_{i j t}\right) \geq \gamma_{1}(1+ \\
& \left.\sum_{k \in N: k \neq i, j} \frac{P_{k} / N_{r}}{L_{b}(k, j)} v_{k t}\right), \forall(i, j) \in A, \forall t \in T \\
& x_{i j t} \in\{0,1\}, \forall(i, j) \in A, \forall t \in T, \\
& v_{i t} \in\{0,1\}, \forall i \in N, \forall t \in T \\
& y_{t} \in\{0,1\}, \forall t \in T
\end{aligned}
$$

Constraints (12) and (13) correspond to (4) and (5), respectively. Constraints (14) state that two adjacent links must be assigned different time slots. Variables $x$ are coupled to variables $v$ using (15), and these two sets of variables are then used in the SIR-constraint (16). Similar observations, as for NSP, concerning the coefficients $M_{i j}$ can be made for LSP. Moreover, for breaking symmetry, we may add $y_{t} \leq y_{t-1}, t=2, \ldots,|T|$, and set $x_{i j t}=0$ if the link index of $(i, j)$ is less than $t$.

\section{Set Covering Formulations}

Formulations NSP and LSP are quite straightforward, but not efficient from a computational point of view. Even for a network of moderate size, the numbers of variables and constraints become very large. In our numerical experiments, the state-of-the-art mixed integer solver CPLEX (version 7.0) is only able to solve the smallest instance (a network with 20 nodes) of NSP to optimality. For LSP, CPLEX did not manage to find a feasible integer solution for any of our instances within reasonable time.

Instead of using formulations NSP and LSP, in this section we reformulate the two optimization problems using set covering formulations, for which a column generation approach can be used for solving the linear programming relaxations. This approach is similar to the one used in [15] for solving graph coloring problems.

To obtain set covering formulations, we consider a group of nodes (or links) that can transmit simultaneously. Such a group defines a column in the set covering formulations. To proceed, we let $L_{N}$ and $L_{A}$ denote the sets of all feasible groups of nodes and links, respectively, and define the following variables related to the groups (or columns).

$x_{l}= \begin{cases}1 & \text { if column } l \text { is used } \\ 0 & \text { otherwise. }\end{cases}$

For node assignment and link assignment, respectively, let

$s_{i l}= \begin{cases}1 & \text { if column } l \text { contains node } i, \\ 0 & \text { otherwise, }\end{cases}$

and

$s_{i j l}= \begin{cases}1 & \text { if column } l \text { contains link }(i, j), \\ 0 & \text { otherwise. }\end{cases}$

The set covering formulation for node assignment (NSCP) is stated below.

$$
\begin{aligned}
{[\mathrm{NSCP}] } & \min \sum_{l \in L_{N}} x_{l} \\
& \sum_{l \in L_{N}} s_{i l} x_{l} \geq 1, \forall i \in N, \\
& x_{l} \in\{0,1\}, \forall l \in L_{N} .
\end{aligned}
$$

The objective is to minimize the number of columns used, and the constraints (21) state that every node is covered by at least one column. Below is the corresponding formulation for link assignment. 


$$
\begin{aligned}
\text { [LSCP] } & \min \sum_{l \in L_{A}} x_{l} \\
& \sum_{l \in L_{A}} s_{i j l} x_{l} \geq 1, \forall(i, j) \in A, \\
& x_{l} \in\{0,1\}, \forall l \in L_{A} .
\end{aligned}
$$

NSCP and LSCP have a very simple constraint structure. Note that the complexity of the problems lies mainly in the construction of the sets $L_{N}$ and $L_{A}$. For realistic problem instances, the sizes of these sets are very large. It is therefore not practical to generate all the columns in the two sets a priori. To overcome this difficulty, we apply a column generation solution approach.

\section{Column Generation}

NSCP and LSCP have, for realistic problem instances, far too many columns to be handled directly by an integer programming method, and the same holds for the corresponding LP-relaxations. Column generation of the LPrelaxation provides a decomposition of the problem into master and subproblems. In this approach, columns are left out because there are too many to handle efficiently, and most of them will not be used in an optimal solution anyway. To check optimality of the LP-relaxation, a subproblem, called the pricing problem, which is a separation problem for the dual LP, is solved in order to identify new columns to enter the basis. If such columns are found, the LP is reoptimized. A classical application of the column generation technique is the cutting stock problem, first presented in [7] and [8].

To solve the integer problem, branching is needed when no columns price out to enter the basis and the LP-solution does not satisfy the integrality conditions. Branch-and-price allows column generation to be applied throughout the branch-and-bound tree - and this must be done - in order to guarantee an optimal integer solution. For NSCP and LSCP, we can apply a branch-and-price scheme, similar to that in [15], in order to ensure integer optimality. However, in our computational experiments we found that the LP-optimum was equal, or very close, to the integer optimum. In addition, the columns needed to solve the LP-relaxation were sufficient to enable optimal or near-optimal integer solutions.

\section{A. Node Assignment}

The master problem for node assignment (NAMP), is the LP-relaxation of NSCP with respect to a subset of columns, $L_{N}^{0} \subseteq L_{N}$. Initially, $L_{N}^{0}$ can be the set of columns where each column contains one single node. (This, in fact, corresponds to conventional TDMA.)

$$
\begin{aligned}
& {[\mathrm{NAMP}] \quad \min \sum_{l \in L_{N}^{0}} x_{l}} \\
& \sum_{l \in L_{N}^{0}} s_{i l} x_{l} \geq 1, \forall i \in N, \\
& 0 \leq x_{l} \leq 1, \quad \forall l \in L_{N}^{0} .
\end{aligned}
$$

When NAMP is solved, we need to identify new columns to enter the basis, or possibly verify optimality, by examining whether any of the variables, $x_{l}, l \in L_{N} \backslash L_{N}^{0}$, has a negative reduced cost. This can be done by minimizing the reduced cost $\bar{c}_{l}$ for all the columns in the set $L_{N} \backslash L_{N}^{0}$. The reduced cost of column $l$ can be expressed as $\bar{c}_{l}=1-\sum_{i \in N} \bar{\beta}_{i} s_{i l}$, where $\bar{\beta}_{i}, \forall i \in N$, are the optimal dual variables to (27). The minimum reduced cost can then be expressed as

$$
\min _{l \in L_{N} \backslash L_{N}^{0}} \bar{c}_{l}=1-\max _{l \in L_{N} \backslash L_{N}^{0}} \sum_{i \in N} \bar{\beta}_{i} s_{i l} .
$$

To solve (29), we formulate the column generation subproblem for node assignment (NASUB). We introduce the following variables.

$s_{i}= \begin{cases}1 & \text { if node } i \text { is included in the column, } \\ 0 & \text { otherwise. }\end{cases}$

NASUB can then be formulated as

$$
\begin{aligned}
& \text { [NASUB] } \max \sum_{i \in N} \bar{\beta}_{i} s_{i} \\
& s_{i}+\sum_{j:(j, i) \in A} s_{j} \leq 1, \forall i \in N, \\
& \\
& \frac{P_{i} / N_{r}}{L_{b}(i, j)} s_{i}+\gamma_{1}\left(1+M_{i j}\right)\left(1-s_{i}\right) \geq \\
& \\
& \gamma_{1}\left(1+\sum_{k \in N: k \neq i, j} \frac{P_{k} / N_{r}}{L_{b}(k, j)} s_{k}\right), \forall(i, j) \in A, \\
& s_{i} \in\{0,1\}, \forall i \in N .
\end{aligned}
$$

If the optimal solution to NASUB results in a reduced cost that is non-negative, the optimal LP-value to NSCP is found. Otherwise, NAMP is reoptimized with a new column added to $L_{N}^{0}$, and the procedure continues. At termination, the LP solution value is a lower bound to the integer optimum of NSCP.

\section{B. Link Assignment}

Similar to NSCP, the LP-relaxation of LSCP can be solved by column generation. The corresponding master problem (LAMP), with a subset of columns $L_{A}^{0} \subseteq L_{A}$, can be formulated as follows.

$$
[\mathrm{LAMP}] \min \sum_{l \in L_{A}^{0}} x_{l}
$$




$$
\begin{aligned}
& \sum_{l \in L_{A}^{0}} s_{i j l} x_{l} \geq 1, \forall(i, j) \in A \\
& 0 \leq x_{l} \leq 1, \quad l \in L_{A}^{0} .
\end{aligned}
$$

To find the minimum reduced cost among all neglected columns, we want to find

$$
\min _{l \in L_{A} \backslash L_{A}^{0}} \bar{c}_{l}=1-\max _{l \in L_{A} \backslash L_{A}^{0}} \sum_{(i, j) \in A} \bar{\beta}_{i j} s_{i j l},
$$

where $\bar{\beta}_{i j}, \forall(i, j) \in A$, are the optimal dual variables to (35). This leads to the following column generation subproblem (LASUB),

$$
\begin{aligned}
& \text { [LASUB] } \max \sum_{(i, j) \in A} \bar{\beta}_{i j} s_{i j} \\
& \qquad \sum_{j:(i, j) \in A} s_{i j}+\sum_{j:(j, i) \in A} s_{j i} \leq 1, \forall i \in N, \\
& s_{i j} \leq v_{i}, \forall(i, j) \in A, \\
& \frac{P_{i} / N_{r}}{L_{b}(i, j)} s_{i j}+\gamma_{1}\left(1+M_{i j}\right)\left(1-s_{i j}\right) \geq \\
& \gamma_{1}\left(1+\sum_{k \in N: k \neq i, j} \frac{P_{k} / N_{r}}{L_{b}(k, j)} v_{k}\right), \forall(i, j) \in A, \\
& s_{i j} \in\{0,1\}, \forall(i, j) \in A \\
& v_{i} \in\{0,1\}, \forall i \in N,
\end{aligned}
$$

where the variables have the following definitions.

$$
\begin{aligned}
& s_{i j}= \begin{cases}1 & \text { if link }(i, j) \text { is included in the column, } \\
0 & \text { otherwise }\end{cases} \\
& v_{i}= \begin{cases}1 & \text { if node } i \text { is transmitting, } \\
0 & \text { otherwise }\end{cases}
\end{aligned}
$$

As for the node assignment case, we obtain a lower bound to LSCP when column generation terminates.

\section{NUMERICAL RESULTS}

We have used three networks obtained from the Swedish Defense Research Agency. The networks contain 20, 40, and 60 nodes, respectively. The numbers of links are 134, 184, and 396. The network with 20 nodes is shown in Section III.

We use CPLEX (version 7.0) to solve the master problems (NAMP and LAMP) in the column generation method. In order to find integer feasible solutions, the integer versions of NAMP and LAMP are solved to optimality by CPLEX when column generation terminates.

\section{A. Node Assignment}

CPLEX was used to solve the node-slot formulation, NSP, of these instances. For the network with 20 nodes, CPLEX found the optimal solution with 16 time slots, in a solution time of 1 second. For the other two networks, however, CPLEX did not manage to find or to verify the
TABLE I

NUMERICAL RESULTS OF COLUMN GENERATION FOR NODE ASSIGNMENT.

\begin{tabular}{lrrrrr}
\hline Network & LP & IP & Iterations & Time & Reuse \\
\hline N20 & 16 & 16 & 10 & $3 \mathrm{~s}$ & 1.25 \\
N40 & 14 & 15 & 43 & $32 \mathrm{~s}$ & 2.86 \\
N60 & 26 & 26 & 60 & $271 \mathrm{~s}$ & 2.31 \\
\hline
\end{tabular}

optimal solutions within the time limit, which was set to 10 hours. At termination, the best integer solutions use 15 and 29 time slots, respectively.

The results of the column generation method are summarized in Table I, where LP shows the optimal value of the LP-relaxation, and IP is the objective value of the optimal integer solution of NAMP with respect to all the columns needed for solving the LP-relaxation. The next three columns in the table show the number of iterations, the solution time, and the degree of spatial reuse, respectively. The spatial reuse is defined as the ratio between the number of time slots needed in conventional TDMA and the number of time slots in the our integer solution. For node assignment, the former equals $|N|$.

For the networks with 20 and 60 nodes, we are able to find and verify the integer optimal solution. For the network with 40 nodes, the integer solution and the LPbound differs by only one time slot.

\section{B. Link Assignment}

For the same network, the size of LSP is considerably larger than that of NSP. For none of the LSP instances, CPLEX was able to find a feasible integer solution within reasonable time. A direct application of column generation also failed for the networks with 40 and 60 nodes, because of the very long time (up to hours) needed for solving the subproblem LASUB to optimality. We have therefore made the following modification to the column generation method. Instead of solving the subproblem to optimality (which yields the column with the most negative reduced cost), we halt the branch-and-bound enumeration for the subproblem when two integer feasible solutions have been found. If the reduced cost (of the incumbent) is less than or equal to a threshold value of -0.1 , we terminate the solution process for the subproblem, and add the incumbent column to the master problem LAMP. Otherwise, we resume the branch-and-bound enumeration, which is halted again when another integer solution is found, and so on. Solving the subproblem in this fashion, we terminate the enumeration either when a column with the reduced cost less than or equal to the threshold value is found, or when it is verified that no column with negative reduced cost exists.

With the above modification, the column generation method was able to solve all the three instances. The results are displayed in Table II. 
TABLE II

NUMERICAL RESULTS OF COLUMN GENERATION FOR LINK ASSIGNMENT.

\begin{tabular}{lrrrrr}
\hline Network & LP & IP & Iterations & Time & Reuse \\
\hline N20 & 70 & 70 & 175 & $262 \mathrm{~s}$ & 1.91 \\
N40 & 43 & 45 & 360 & $947 \mathrm{~s}$ & 4.09 \\
N60 & 114 & 115 & 874 & $10936 \mathrm{~s}$ & 3.44 \\
\hline
\end{tabular}

We observe that the lower bounds provided by the LPrelaxation are very tight in all three cases. For one network the integer optimum is found. For the other two networks the integer solutions use one and two time slots more than the LP-bounds, respectively.

\section{CONCLUSIONS}

We have addressed resource optimization in ad hoc networks using STDMA. In particular, we consider the problems of assigning time slots for node- and link-oriented assignment strategies. We show NP-hardness results for the two problems, and present two linear integer formulations. These two formulations are, as shown by our numerical experiments, not efficient from the computational point of view. To enable an efficient solution method, we develop set covering formulations for the problems. Our numerical results show that the LP-relaxations of the set covering formulations can be efficiently solved using a column generation approach, and that they provide very tight bounds to the integer problems. In addition, column generation enables integer solutions that are very close to the theoretical LP-bounds. That our approach can be used to generate valuable benchmarks for other heuristic algorithms is, therefore, the main contribution of this work.

There are many remaining tasks and challenges for future research. One issue to be addressed is a further validation of the proposed method, by extending the computational experiments for other network topologies and instances. The next important step of our research is to consider more complex problems, for example, resource optimization that takes traffic distribution into account. In particular, we note that the methodology developed in this paper can be extended to provide benchmarks when maximizing the network throughput. We also plan to investigate whether our method is helpful in studying capacity regions of ad hoc networks [19]. We will then move into two research directions. First, there is, from a practical point of view, an interest to move from centralized algorithms to distributed ones. Secondly, we need to explicitly consider the time-varying properties of the medium, that is, to tackle mobile scenarios in which the input data is dynamic due to the movements of the radio units. For these complex problems, we will focus on simple but fast heuristics which do not necessarily guarantee optimal solutions. However, the optimal solutions for the basic scenarios, such as those presented in this paper, serve as benchmarks for future research in the area. Having access to these solutions will certainly help in developing heuristic methods for the complex problems.

\section{ACKNOWLEDGMENT}

The authors wish to thank the research group at the Department of Communication Systems, Swedish Defense Research Agency (FOI), for the technical discussions and the test data. This work is partially financed by CENIIT (Center for Industrial Information Technology), Linköping Institute of Technology, Sweden.

\section{REFERENCES}

[1] P. Björklund, Two Resource Optimization Applications in Wireless Networks, Licentiate thesis, Department of Science and Technology, Linköping university, Sweden, 2002.

[2] I. Cidon and M. Sidi, "Distributed assignment algorithms for multi-hop packet radio networks," IEEE Transactions on Computers, vol. 38, pp 1353-1361, 1989.

[3] I. Chlamtac and S. S. Pinter, "Distributed nodes organization algorithm for channel access in a multihop dynamic radio network," IEEE Transactions on Computers, vol. 36, pp 728-737, 1987.

[4] A.-M. Chou and V. O. K. Li, "Slot allocation strategies for TDMA protocols in multihop packet radio networks," Proceedings of IEEE INFOCOM '92, pp 710-716, 1992.

[5] A. Ephremides and T. Truong, "Scheduling broadcasts in multihop radio networks," IEEE Transactions on Communications, vol. 38, pp 456-460, 1990.

[6] N. Funabiki and Y. Takefuji, "A parallel algorithm for broadcast scheduling problems in packet radio networks," IEEE Transactions on Coтmunications, vol. 41, pp 828-831, 1993.

[7] P. C. Gilmore and R. E. Gomory, "A linear programming approach to the cutting stock problem," Operations Research, vol. 9, pp 849-859, 1961.

[8] P. C. Gilmore and R. E. Gomory, "A linear programming approach to the cutting stock problem - part II," Operations Research, vol. 11, 1963.

[9] A. J. Goldsmith and S. B. Wicker, "Design challenges for energyconstrained ad hoc wireless networks," IEEE Transactions on Wireless Communications, pp 8-27, August 2002.

[10] J. Grönkvist, "Traffic controlled spatial reuse TDMA in multi-hop radio networks," Proceedings of 9th IEEE International Symposium on Personal, Indoor and Mobile Radio Communications, pp 1203-1207, 1998.

[11] J. Grönkvist, "Assignment methods for spatial reuse TDMA," Proceedings of IEEE Annual Workshop on Mobile and Ad Hoc Networking and Computing (MobilHOC), pp 119-124, 2000.

[12] J. Grönkvist, A. Hansson and J. Nilsson, "A comparison of access methods for multi-hop ad hoc radio networks," IEEE Vehicular Technology Conference Proceedings, pp 1435-1439, 2000.

[13] J. Grönkvist, Assignment Strategies for Spatial Reuse TDMA, Licentiate thesis, Royal Institute of Technology, Stockholm, Sweden, 2002.

[14] B. Hajek and G. Sasaki, "Link scheduling in polynomial time," IEEE Transactions on Information Theory, vol. 34, pp 910-917, 1988.

[15] A. Mehrotra and M. A. Trick, "A column generation approach for graph coloring," INFORMS Journal on Computing, vol. 8, pp 344-354, 1996.

[16] R. Nelson and L. Kleinrock, "Spatial-TDMA: A collision-free multihop channel access control," IEEE Transactions on Communications, vol. 33 , pp 934-944, 1985.

[17] C. G. Prohazka, "Decoupling link scheduling constraints in multihop packet radio networks," IEEE Transactions on Computers, vol. 38, pp 455-458, 1989.

[18] D. S. Stevens and M. H. Ammar, "Evaluation of slot allocation strategies for TDMA protocols in packet radio networks," Proceedings of the 1990 Military Communications Conference (MILCOM), pp 835-839, October 1990.

[19] S. Toumpis and A. J. Goldsmith, "Capacity regions for wireless ad hoc networks," IEEE International Conference on Communications, pp 3168-3173, 2002. 